\title{
Control of electrical turbulence by periodic excitation of cardiac tissue
}

\author{
Pavel Buran, ${ }^{1}$ Markus Bär, ${ }^{1}$ Sergio Alonso, ${ }^{2}$ and Thomas Niedermayer ${ }^{1, a)}$ \\ ${ }^{1}$ Physikalisch-Technische Bundesanstalt (PTB), Abbestr. 2-12, 10587 Berlin, Germany \\ ${ }^{2}$ Department of Physics, Universitat Politècnica de Catalunya, Av. Dr. Marañón 44, 08028 Barcelona, Spain
}

(Received 20 April 2017; accepted 26 October 2017; published online 17 November 2017)

\begin{abstract}
Electrical turbulence in cardiac tissue is associated with arrhythmias such as life-threatening ventricular fibrillation. Recent experimental studies have shown that a sequence of low-energy electrical far-field pulses is able to terminate fibrillation more gently than a single high-energy pulse which causes severe side effects. During this low-energy antifibrillation pacing (LEAP), only tissue near sufficiently large conduction heterogeneities, such as large coronary arteries, is activated. In order to optimize LEAP, we performed extensive simulations of cardiac tissue perforated by blood vessels, employing two alternative cellular models that exhibit electrical turbulence at a similar length scale. Moreover, the scale of blood vessels in our two-dimensional simulations was chosen such that the threshold for single pulse defibrillation matches experimental values. For each of the 100 initial conditions, we tested different electrical field strengths, pulse shapes, numbers of pulses, and periods between the pulses. LEAP is successful for both models, albeit with substantial differences. One model exhibits a spectrum of chaotic activity featuring a narrow peak around a dominant frequency. In this case, the optimal period between low-energy pulses matches this frequency and LEAP greatly reduces the required energy for successful defibrillation. For pulses with larger energies, the system is perturbed such that underdrive pacing becomes advantageous. The spectrum of the second model features a broader peak, resulting in a less pronounced optimal pacing period and a decreased energy reduction. In both cases, pacing with five or six pulses which are separated by the dominant period maximizes the energy reduction. Published by AIP Publishing.

https://doi.org/10.1063/1.5010787
\end{abstract}

In ventricular fibrillation, the heart is quivering instead of pumping, a condition which leads to cardiac arrest and ultimately to death. This loss of synchronous contraction stems from disorganized electrical activity in the ventricles. Emergency defibrillation is achieved by the application of a strong electrical shock which is accompanied by severe side effects such as tissue damage and trauma. Recently, an alternative treatment using a sequence of low energy pulses has been suggested in which each electrical pulse excites only localized tissue sites at the large heterogeneities-such as blood vessels-instead of the entire tissue. In laboratory experiments in vitro and in vivo, this so-called low-energy antifibrillation pacing (LEAP) with five pulses achieves defibrillation with an energy reduction of $\mathbf{8 0} \%-90 \%$ per pulse in comparison with standard single shock treatment. Here, we have performed an extensive numerical study of LEAP employing far field pacing at the major blood vessels. We have tested two alternative electrophysiological models that exhibit qualitatively different spatiotemporally chaotic activities. Both models exhibit successful LEAP with, however, different success probabilities. The optimal LEAP protocol consists of pulses which are separated by the dominant period of fibrillation. Pacing with a frequency which is similar, but somewhat different from the dominant frequency, as typically done in experiments, results in a

Note: Paper submitted as part of the September 2017 focus issue: Complex Cardiac Dynamics (Guest Editors: Elizabeth M. Cherry, Flavio H. Fenton, Trine Krogh-Madsen, Stefan Luther, and Ulrich Parlitz).

${ }^{a)}$ Electronic mail: niedermayerthomas@gmail.com considerably smaller energy reduction. Our results may provide a link between the spectrum of the electrical activity and the optimal pacing frequency in experimental defibrillation procedures.

\section{INTRODUCTION}

The nonlinear dynamics of electrical excitation waves in cardiac tissue has been studied extensively in experiment and simulation for more than two decades; for recent reviews, see, e.g., Refs. 1-5. A loss of rhythm and synchronization of cardiac electrical impulses orchestrating heart contraction and pumping of blood is associated with a number of arrhythmias including atrial fibrillation (AF) and ventricular fibrillation (VF). VF represents a particularly dangerous malfunction of the heart, potentially causing sudden death if left untreated for more than a few minutes. The electrical activity during VF is dominated by either one or a few large rotors (mother rotors) surrounded by irregular activity or entirely by multiple wavelets in a spatiotemporally chaotic state. First, direct experimental observations of such states were reported almost 20 years ago. ${ }^{6,7}$ Recent experimental and simulations studies ${ }^{8,9}$ show a complex picture with states dominated by one or a few mother rotors as well as multiple wave dynamics characterized by a mean number of 10 vortices on the surface of, e.g., a human heart. The fluctuations in the vortex number resemble a Poissonian statistics. 
Defibrillation by a strong electrical pulse is the only effective therapy for VF, readily used in clinics. Such a strong shock globally excites the tissue resulting in termination of all excitation waves but also causes severe side effects as pain and trauma for the patient as well as, ${ }^{10}$ damage of the myocardium. ${ }^{11}$ These adverse effects could be diminished if VF could be terminated reliably by defibrillation shocks with significantly lower energies. Recent studies $^{12,13}$ of $\mathrm{AF}$ in vitro and in vivo as well as $\mathrm{VF}$ in vitro have shown that a sequence of five low-energy far-field pulses with stimulation rates close to the arrhythmia cycle length can require $80 \%-90 \%$ less energy per pulse than a single shock. This method is called low-energy antifibrillation pacing (LEAP). With much faster pacing rates than the cycle length, similar energy reductions were found for $\mathrm{AF}^{14,15}$ while for ventricular tachycardia (VT), the energy may further be reduced by an order of magnitude. ${ }^{16,17}$

LEAP takes advantage of the fact that an electric field depolarizes and hyperpolarizes the tissue near conductivity heterogeneities ${ }^{18}$ which become virtual electrodes. ${ }^{19}$ The strength of this effect depends on the size and shape of the heterogeneities and the strength of the electric field. ${ }^{20-22}$ Only tissue at major conduction heterogeneities may be activated by each of these very weak pulses. Therefore, global tissue activation and wave termination originate from few localized activation sites (hot spots). The mechanism how multiple low-energy far-field pulses terminate arrhythmias is not well understood as experimental methods to visualize three dimensional electrical turbulence are missing. Luther et al. ${ }^{13}$ suggest that the major conduction heterogeneities necessary for LEAP are given by large coronary arteries and show that the size distribution of the heterogeneities follows a power law. However, Caldwell et $a l^{23}$ did not find such a co-localization of hot spots and major coronary vessels.

Theoretical approaches to understand LEAP have often focussed on the process of unpinning and removal of a small number of vortices. ${ }^{20,24-26}$ In these papers, it was demonstrated that vortices that were assumed to be pinned at larger heterogeneities in cardiac tissue are best terminated if the pacing frequency in a LEAP procedure is set to be $80 \%-90 \%$ of the dominant frequency (the rotation frequency of the vortex around the pinning site). Such a choice of pacing frequency allows for an efficient scanning of the phase of the vortex in order to find the vulnerable window for vortex termination. ${ }^{27,28}$ A very different simulation study in a realistic three-dimensional cardiac geometry demonstrated for selected examples that LEAP works for a pacing period of $88 \%$ of the VF cycle length but often fails for much faster pacing with a period of $16 \%$ of the VF cycle length. ${ }^{29}$

In LEAP experiments, reduction of defibrillation energy is the quantity which is studied in the quasi-two-dimensional $\mathrm{AF}$ and the three dimensional VF. ${ }^{13}$ Very similar results are found in both cases and, moreover, the size distribution of the radii of arteries is found to be almost identical for atria and ventricles. Therefore, we expect that LEAP can be qualitatively investigated within a simple two dimensional model in which the non-conducting heterogeneities are represented by circles whose size distribution is given by the distribution of radii of coronary arteries measured in Ref. 13. Furthermore, we tested three alternative models which are established for ventricular cells $\mathrm{s}^{30-32}$ and studied two of these in detail ${ }^{30,31}$ while the other ${ }^{32}$ exhibits intricacies which require careful investigations in another study. Importantly, we have rescaled the alternative cellular models such that they exhibit identical length scales of electrical turbulence. Together with the scale of arterial radii, which is obtained from measurements, these scales result in the correct magnitude for the electric field of single pulse defibrillation. The simplicity of our composite model allows us to perform systematic statistical studies with a set of 100 initial conditions. This is essential since both standard defibrillation and LEAP may depend critically on initial conditions and are also associated with large uncertainties. ${ }^{13}$

In our computational study, we aim for a basic understanding of LEAP to potentially optimize its protocol. Therefore, we have investigated many aspects of LEAP which might be crucial for its success. It turns out that a successful LEAP protocol must posses certain properties of the electrical turbulence to be terminated. Moreover, we found a LEAP protocol which is even more efficient in our simulations than the one employed in experimental studies.

In Sec. II, we introduce the cellular models in a monodomain framework, the distribution of heterogeneities, the alternative defibrillation protocols, the numerical methods used in the simulations, and our choice of initial conditions. Sec. III contains the main results regarding characterization of electrical turbulence in the two models, single pulse defibrillation and LEAP as well as the survival statistics of fibrillation activity in the absence of defibrillation. Sections IV and $\mathrm{V}$ provide a discussion of the results and a short conclusion, respectively.

\section{METHODS}

We have performed extensive numerical simulations with two cellular models and defibrillation protocols with different electrical field strengths, wave forms, numbers of pulses, and inter-pulse intervals. Typically, 100 simulation runs with different initial conditions were performed for each configuration. To obtain statistically valid results, we restricted the simulations to a homogeneous, isotropic, and two-dimensional tissue which is perforated by blood vessels.

\section{A. Mono-domain equation and scaled models}

By homogenization, cardiac tissue is represented by intra-cellular and extra-cellular space. ${ }^{5,33}$ Assuming vanishing anisotropy, the propagation of electrical waves is described by the mono-domain equation ${ }^{33}$

$$
\partial_{t} V(\vec{x}, t)=D \Delta V(\vec{x}, t)-\frac{I_{\text {ion }}(V(\vec{x}, t), \vec{w}(\vec{x}, t))}{C},
$$

where $V$ denotes the transmembrane potential, $\Delta$ the Laplacian, $C$ the specific membrane capacitance, and $I_{\text {ion }}$ the sum of transmembrane current densities which is a function of both the transmembrane potential $V$ and several state variables $\vec{w}$. The (diffusion) coefficient $D$ is determined by tissue 
conductivity, membrane capacitance, and the area to volume ratio of the cellular model. Experimental studies have shown that the magnitude of the pulse-induced transmembrane potential saturates with increasing electric field strength due to electroporation. ${ }^{34}$ Therefore, we limit the minimum and maximum value of the transmembrane potential to $-150 \mathrm{mV}$ and $+150 \mathrm{mV}$, respectively.

The dynamics of the state variables $\vec{w}$ in Eq. (1) is specified by a particular cellular model. To our knowledge, reliable information about the structure of electrical turbulence during ventricular fibrillation is lacking, as cellular models are built to reproduce the activity in single cells and even recent models of human ventricular cells exhibit a very diverse spatiotemporal behavior. ${ }^{35}$ Thus, we tested three cellular models which exhibit qualitatively different spatiotemporal chaos and investigated two of these in detail: The Luo-Rudy (LR) model with standard parameter values ${ }^{30}$ and the Fenton-Karma (FK) model $^{31}$ with $V_{0}=-84 \mathrm{mV}, V_{\text {fi }}=23.125 \mathrm{mV}$, and the default parameter values in Ref. 36 which maximize the propensity for spiral waves to break while minimizing core meandering. A few explorative simulations of the Ten Tusscher model ${ }^{32}$ with parameter values matching human epicardial tissue did not give conclusive results; thus. we adjourn the systematic investigation of this model to another study.

The specific membrane capacitance $C$ in Eq. (1) depends on the specific cardiac tissue considered. We use the estimate $C=1 \mu \mathrm{F} / \mathrm{cm}^{2}$, which is similar to the measured value in trabecular muscle of mammalian heart. ${ }^{37}$ The value of the diffusion coefficient $D$ is largely unknown. Often, it is adjusted in simulation studies, see for instance Ref. 29. Alternatively, one may estimate it from properties of cardiac cells. For human ventricular cells, $D=(1.171 \pm 0.221) \times 10^{-4} \mathrm{~m}^{2} / \mathrm{s}$ has been calculated. ${ }^{38}$

For simulations of the (LR) model, we adapt the order of magnitude and use $D_{\mathrm{LR}}=10^{-4} \mathrm{~m}^{2} / \mathrm{s}$. Tentatively, we have chosen $20 \mathrm{~cm} \times 20 \mathrm{~cm}$ for the extension of our $2 \mathrm{~d}$ tissue which is the same order of magnitude as the surface of a (large) mammalian heart. More importantly, with this system size, the LR model ensures the correct orders of magnitude for two (system size dependent) properties of fibrillation/ electrical turbulence: About ten phase defects, ${ }^{8}$ see Fig. 1(b), and a half-life of turbulence which does not fall below $9 \mathrm{~s}$ for periodic boundary conditions, see Fig. 3. Therefore, we interpret the extension of our $2 \mathrm{~d}$ system less in terms of tissue size but more in terms of the effective system size. Consequently, we specify the diffusion constant for the (LR) model in the form $D_{\mathrm{LR}}=0.2 \xi^{2} / \mathrm{s}$, where $\xi$ is the spatial correlation length, see Fig. 1(a). For simulations of the (FK) model, we have chosen $D$ such that the correlation length $\xi$ during electrical turbulence is identical to the one found in the (LR) model. This results in $D_{\mathrm{FK}}=3.13 \xi^{2} / \mathrm{s}$ or $D_{\mathrm{FK}}$ $\approx 1.6 \times 10^{-3} \mathrm{~m}^{2} / \mathrm{s}$ assuming the original extension of the system. In consequence, we again find about 10 rotors, see Fig. 1(c), but an increased half-life of turbulence, see Fig. 3.

\section{B. Blood vessels as heterogeneities}

Blood vessels constitute large heterogeneities of conductivity ${ }^{13}$ and were modeled as non-conducting patches in otherwise homogenous tissue. The intracellular current into these heterogeneities is zero, and in the presence of an external electric field $\vec{E}$, this leads to the boundary condition $(\nabla V-\vec{E}) \cdot \vec{n}=0,{ }^{20,22}$ where $\vec{n}$ is normal to the boundary of the heterogeneity. In consequence, an external electric field increases the transmembrane potential in the vicinity of one side of the heterogeneity and may activate this tissue. ${ }^{20,22}$ In general, smaller heterogeneities require a larger field strength for tissue activation. ${ }^{20}$ For a circular heterogeneity in quiescent two-dimensional tissue, the minimal radius required for activation is a well-known, monotonically decreasing function of the magnitude of the applied field. ${ }^{13,20}$ In the absence of conduction heterogeneities and tissue anisotropy, the a)

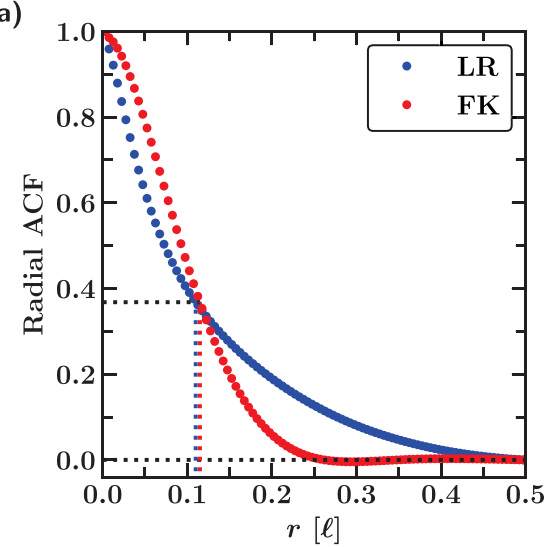

b)

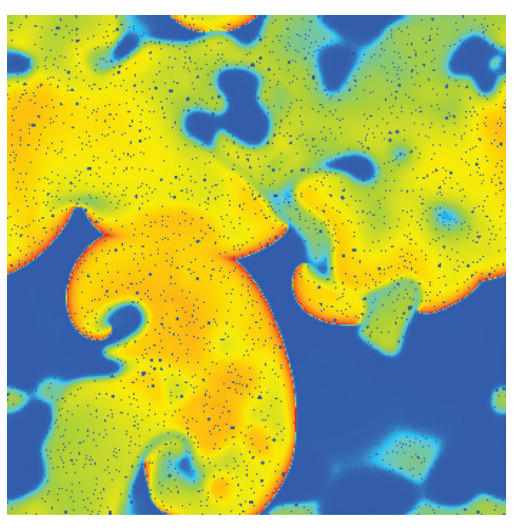

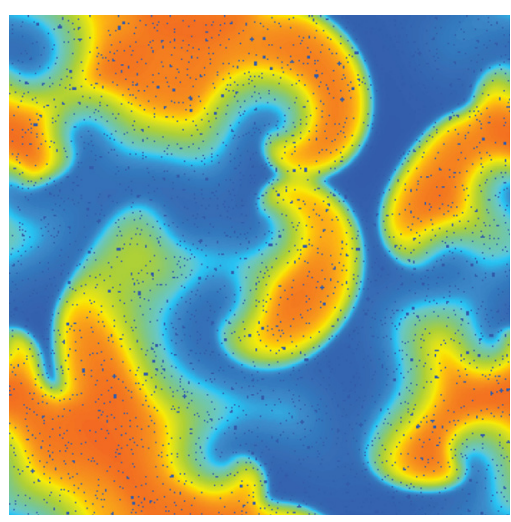

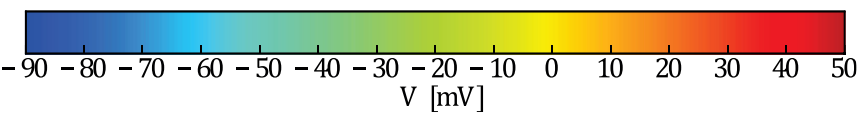

FIG. 1. (a) Radial autocorrelation function of transmembrane voltage $V$ for (LR) and (FK) models. Radial distance $r$ is measured in units of system size $\ell$. We have chosen the diffusion constant $D_{\mathrm{FK}}$ such that the correlation lengths $\xi$, for which $\operatorname{ACF}(\xi)=\operatorname{ACF}(0) e^{-1}$ holds, of both models match. (b) Snapshot of transmembrane potential $V$ during electrical turbulence for the (LR) model. An average number of 10 phase defects are found. (c) Respective snapshot for the (FK) model. Again, an average number of 10 phase defects are found. Simulations were performed on a rectangular domain with an edge length of about $9 \xi$. Conductivity heterogeneities are represented by small blue circles. Movies of the simulations are provided in the supplementary material. 
transmembrane potential is not altered by an external electrical field. ${ }^{33}$

Both in ventricles and in atria of adult beagle dogs, the size distribution of coronary artery radii $R$ is given by a power law ${ }^{13} p(R) \propto R^{\alpha}$. This distribution holds between $R_{\min } \approx 0.06 \mathrm{~mm}$ and $R_{\max } \approx 0.8 \mathrm{~mm}$ and the exponent is given by $\alpha \approx-2.75$ for both ventricles and atria. ${ }^{13}$ Assuming a system dimension of $\ell=20 \mathrm{~cm}$, we modeled coronary arteries in ventricles as circular heterogeneities with radii drawn from this distribution and positions drawn from a uniform distribution while circle overlap is prohibited. For the (area) density of all heterogeneities, $40 / \mathrm{cm}^{2}$ was chosen since this value matches the density of activated arteries in the limit of large $E$, as measured in Ref. 13.

Next, we have tested the thresholds for single pulse defibrillation, see Fig. 4. For both the (LR) and (FK) models, we found values which are consistent with experiments, ${ }^{39}$ indicating that we have chosen the correct scaling between arteries_-assuming these are the major heterogeneities - and the size of turbulent structures, see Fig. 1. Therefore, we may interpret the cutoffs of the distribution as $R_{\min } \approx 2.7 \times 10^{-3} \xi$ and $R_{\max } \approx 3.5 \times 10^{-2} \xi$, and the density of all heterogeneities is given by $400 / \xi^{2}$.

We have checked that different realization of positions and radii resulted in almost identical defibrillation successes. Therefore, the same realization of positions and radii was used in simulations while the initial states of electrical turbulence were varied unless stated otherwise.

\section{Defibrillation protocols}

State-of-the-art defibrillators execute a biphasic, asymmetric protocol. ${ }^{40}$ We tested both monophasic and biphasic defibrillation protocols specified as follows. For monophasic pulses, a rectangular waveform with a duration of $10 \mathrm{~ms}$ and amplitude $E$ was chosen. For biphasic pulses, the waveform consisted of two subsequent rectangular parts with identical amplitudes $E$ and opposite field direction. We fixed the total pulse duration to $10 \mathrm{~ms}$ and optimized the ratio of forward to backward duration. For fixed parameters, we found that $7 \mathrm{~ms}$ (forward) and $3 \mathrm{~ms}$ (backward) lead to the best defibrillation results, in agreement with Refs. 41 and 42, where the best efficiency is obtained when the first phase is about twice as long as the second phase. Our LEAP protocol contains $n$ identical biphasic pulses, where the interval $T$ between two pulses is constant and defined between the onsets of subsequent pulses.

\section{Numerical methods}

The mono-domain Eq. (1) in conjunction with a cellular model was solved on a rectangular, 2-dimensional, and equidistant finite-difference grid with $1000 \times 1000$ nodes, resulting in a grid spacing of $\Delta x=0.2 \mathrm{~mm}$ or $\Delta x \approx 8.85 \times 10^{-3} \xi$. Both periodic and no-flux boundary conditions were used, as discussed below. The diffusion term of Eq. (1) was discretized by the standard five-point stencil. ${ }^{43}$ Since a straightforward explicit Euler time integration requires a rather small time step of $0.001 \mathrm{~ms}$ for numerical stability, we used the Rush-Larsen method $^{44}$ to integrate the gating variables. Furthermore, we considered the diffusion term implicitly, since for the explicit solution, time steps would have been limited by $\Delta x^{2} / 4 D .{ }^{45}$ The resulting linear equation system has a symmetric positive definite coefficient matrix and can be solved efficiently by the conjugate gradient method ${ }^{43}$ These modifications allowed us to increase the time step to $0.1 \mathrm{~ms}$ for the (LR) model and $0.025 \mathrm{~ms}$ for the (FK) model.

\section{E. Generation of initial conditions}

A single spiral wave was initiated using the standard cross-field stimulation technique 46 on a grid with no-flux boundary conditions. We have run the system for $5 \mathrm{~s}$ to allow spiral break and onset of electrical turbulence. Subsequently, the transmembrane potential in 10 randomly chosen squares, with edge lengths of $0.05 \ell$, was set to the excitation threshold, the boundary conditions were changed (periodic or noflux, depending on the simulations performed later), and the systems evolved for one more second to establish different initial conditions. In total, we generated 100 initial states of electrical turbulence (fibrillation) which we used for simulations with (LR) and (FK) cellular models, both with periodic and no-flux boundary conditions.

\section{RESULTS}

\section{A. Electrical turbulence}

After spatial rescaling and generating the initial conditions as discussed above, both the (LR) and (FK) models support electrical turbulence representing fibrillation, see Fig. 1 and movies in the supplementary material. However, the spectra of the transmembrane potential are qualitatively different: The (LR) model exhibits a sharp peak indicating a dominant cycle length of $\tau_{\mathrm{LR}} \approx 0.33 \mathrm{~s}$ while the main peak in the spectrum of the (FK) model is more expanded, see Fig. 2. Moreover, the (LR) spectrum features two additional peaks at about half and double of the dominant cycle length while the (FK) spectrum is rather unstructured.

Previous simulation studies have indicated that spatiotemporal chaos in generic one- and two-dimensional reaction-diffusion systems ${ }^{47-49}$ and electrical turbulence in cardiac tissue $^{36}$ are often transient states which depend on the system size. Therefore, we have rescaled the system as detailed in Sec. II A and checked the lifetimes of electrical turbulence in the absence of defibrillation and with initial conditions generated as described in Sec. II E. With no-flux boundaries, both the (LR) and (FK) models exhibit rather short spatiotemporal chaos, see Fig. 3. Naturally, periodic boundaries increase turbulence lifetimes and we find $t_{1 / 2} \approx 9.2 \mathrm{~s}$ for the half-life of the turbulence in the (LR) model and a considerably larger value for the (FK) model.

\section{B. Single pulse defibrillation}

Running $N=100$ simulations (see supplementary material) for each parameter configuration and starting from different initial conditions as presented above, we determined the single pulse termination probability $P^{\mathrm{S}}$, which is defined as the probability that a fibrillation state is converted into a quiescent state by a single defibrillation pulse, as a function of field 


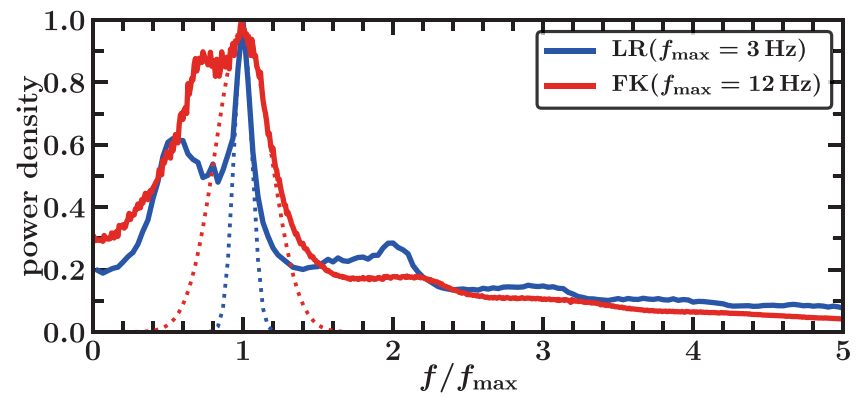

FIG. 2. Scaled spectra of transmembrane potential $V$ during electrical turbulence for the Luo-Rudy ${ }^{30}$ (LR, top) and Fenton-Karma ${ }^{31}$ (FK, bottom) model, respectively. Simulations were performed for $10 \mathrm{~s}$ and with 10 different initial conditions exhibiting electrical turbulence. The displayed graphs are averages of spectra of the transmembrane potential $V$ at all spatial grid points and from all simulation runs. The spectrum of the (LR) model is characterized by a narrow peak at $f_{\max }=3 \mathrm{~Hz}$ and two local maxima at about double and half of this frequency. The (FK) model exhibits a much broader peak. The full widths at half maximum (FWHM) of the displayed Gaussian peaks are given by $\delta f / f_{\max } \approx 0.146$ and $\delta f / f_{\max } \approx 0.44$ for the $(\mathrm{LR})$ and (FK) models, respectively.

strength $E$. We computed $P^{\mathrm{S}}$ as the fraction of successful termination events, see Fig. 4. This is a statistically sound estimate since its error is given by $\delta P^{\mathrm{S}}=\sqrt{P^{\mathrm{S}}\left(1-P^{\mathrm{S}}\right) / N}$ $\sim 1 / \sqrt{N}$. We have checked both mono-phasic and bi-phasic pulse protocols as well as both periodic and no-flux boundary conditions. For a given cellular model and fixed pulse duration, bi-phasic pulses ( $3 \mathrm{~ms}$ forward, $7 \mathrm{~ms}$ backward) are more effective than mono-phasic ( $10 \mathrm{~ms}$ forward) pulses, see Fig. 4. This is in line with earlier findings. ${ }^{42}$ On the other hand, the defibrillation success is almost unaffected by the boundary conditions. We also estimated both the minimum electric field strength $E_{90}^{\text {mono }}$ of a single monophasic pulse and $E_{90}^{\text {bi }}$ of a single biphasic pulse which give rise to termination probability of at least $90 \%$. With periodic boundary conditions, we found $E_{90}^{\text {mono }} \approx 12 \mathrm{~V} / \mathrm{cm}$ and $E_{90}^{\text {bi }} \approx 5.0 \mathrm{~V} / \mathrm{cm}$ for the (LR) model and $E_{90}^{\text {mono }} \approx 4.4 \mathrm{~V} / \mathrm{cm}$ and $E_{90}^{\mathrm{bi}} \approx 3.8 \mathrm{~V} / \mathrm{cm}$ for the $(\mathrm{FK})$ model. Systematic experiments with mono-phasic pulses applied to over 200 fibrillation episodes of canine hearts in vivo are consistent with our results: While defibrillation is hopeless for field strengths below $2.9 \mathrm{~V} / \mathrm{cm}$ and guaranteed for field strengths above $8.5 \mathrm{~V} / \mathrm{cm}$, the regime in-between is characterized by an increasing success probability. ${ }^{39}$ As we have

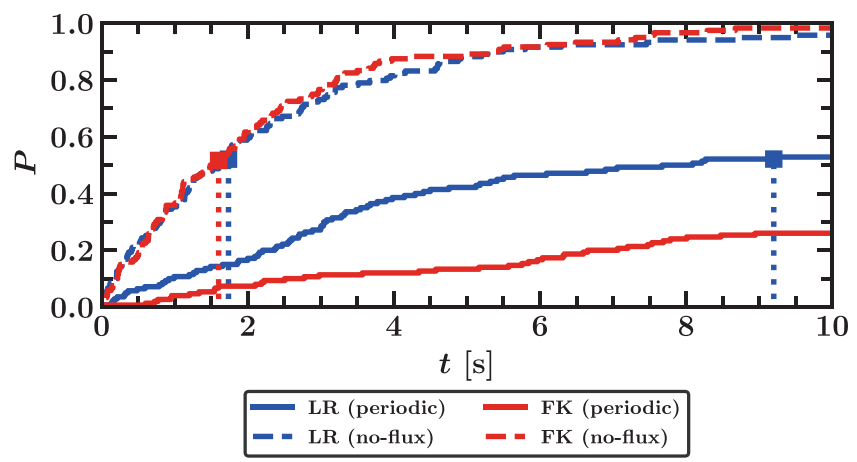

FIG. 3. Lifetimes of electrical turbulence in the absence of defibrillation. Termination probabilities $P$ for the (LR, blue) and (FK, red) model, respectively, with periodic (solid lines) and no-flux boundaries (dashed lines) are shown as functions of time $t$. Dotted vertical lines indicate the half-lifes $t_{1 / 2}$ of transient electrical turbulence.

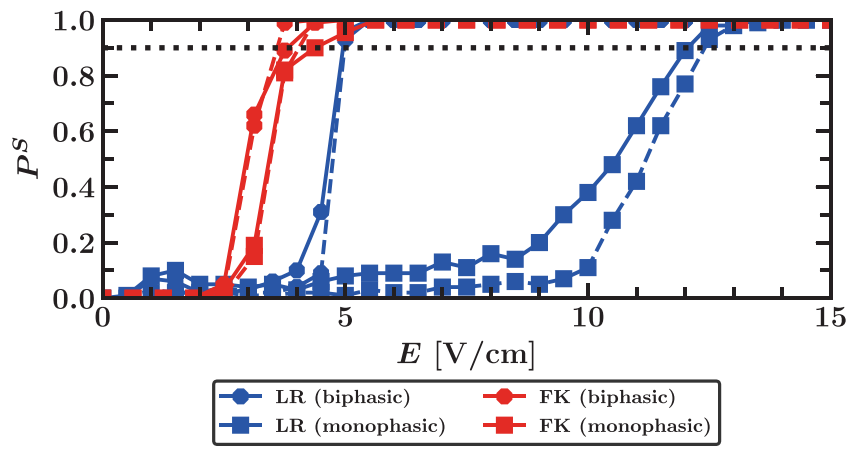

FIG. 4. Single pulse termination probabilities $P^{\mathrm{S}}$ as functions of the applied field strength $E$ for the (LR, blue) and (FK, red) model, respectively, and for monophasic (squares) and biphasic (circles) pulse shapes with $10 \mathrm{~ms}$ duration. Solid lines represent simulations with periodic boundaries. No-flux boundaries (dashed lines) produce almost identical outcomes. The dotted horizontal line indicates a termination probability of $90 \%$ and defines $E_{90}$, i.e., the minimum electric field strength to obtain a termination probability of at least $90 \%$.

discussed in Sec. II B, this consistency ensures the correct scaling between artery sizes and dimensions of turbulent structures which are terminated.

\section{Low-energy antifibrillation pacing (LEAP)}

Since single biphasic pulses are favorable for defibrillation compared to single monophasic pulses, see Sec. III B, we employed the former for LEAP. However, we also noted that for the small field strength employed for LEAP, there is virtually no difference between the efficiencies of mono- and biphasic pulses.

Successful LEAP has been defined by the reduction of energy per pulse of several weak pulses in comparison to a single pulse. ${ }^{13}$ Here, we give a more precise definition, adopting our probabilistic description of termination of turbulence. For finite termination probabilities of individual pulses, the termination probability of a sequence of uncorrelated pulses with given field strength increases with the number of pulses. Accordingly, for a desired termination probability, the required field strength - and thus the energy per pulse-decreases with the number of pulses, resulting in trivially successful LEAP. Therefore, we adopt a stricter definition for successful LEAP by requesting a minimal energy reduction of $50 \%$ per pulse. More precisely, we define LEAP to be successful if $E_{90}^{\mathrm{L}}$ $<E_{90}^{\mathrm{bi}} / \sqrt{2}$, where $E_{90}^{\mathrm{L}}$ is the electric field strength of LEAP to achieve a termination probability of $90 \%$ and $E_{90}^{\text {bi }}$ is the corresponding quantity for biphasic pulses.

With this definition, LEAP is successful in controlling electrical turbulence in cardiac tissue described by the (LR) or (FK) model, see Figs. 5 and 7 and movies in the supplementary material. LEAP in both models exhibits common features and differences. Before discussing these, we report on our result for the (LR) model in detail.

\section{a. LEAP for the (LR) model}

The pulse interval $T$ turns out to be crucial for LEAP success, see Fig. 5a. Maximal termination probability $P^{\mathrm{L}}$ is found for $T_{\max } \approx 330 \mathrm{~ms}$ which matches the dominant period $\tau_{\mathrm{LR}} \approx 330 \mathrm{~ms}$ of the fibrillation state of the (LR) model, see 
a)

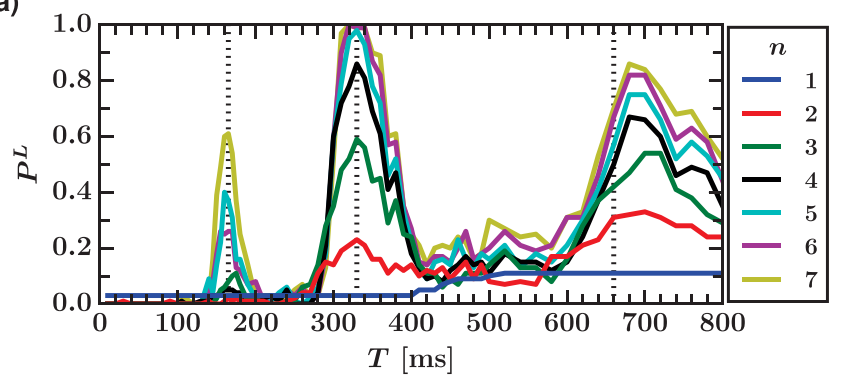

b)

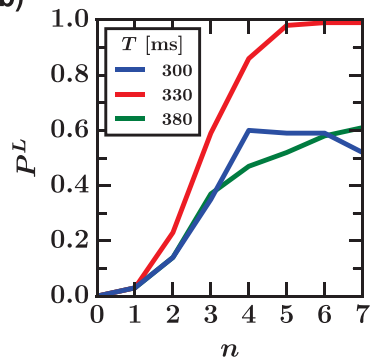

c)

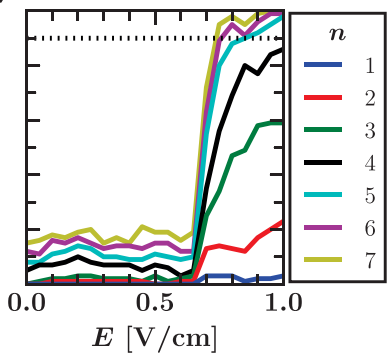

d)

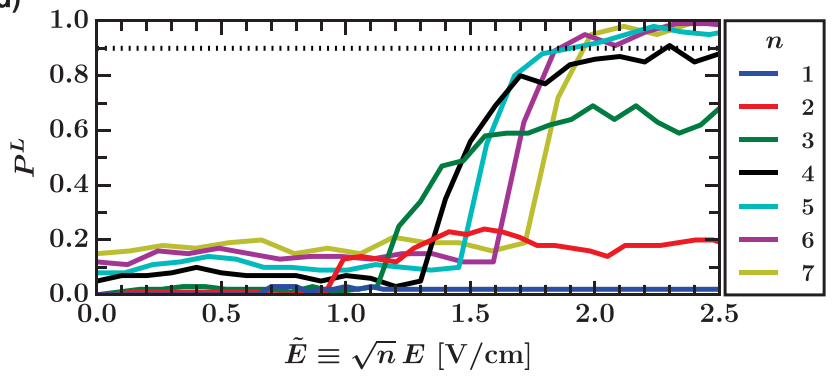

FIG. 5. Termination probability $P^{\mathrm{L}}$ of LEAP for the (LR) model. (a) $P^{\mathrm{L}}$ as a function of pulse interval $T$ for different pulse numbers $n$ and fixed field strength $E=1 \mathrm{~V} / \mathrm{cm}$. For $n \geq 3$, each curve exhibits an absolute maximum at $T_{\max }=330 \mathrm{~ms}$ and pronounced local maxima near $T_{\max } / 2$ and $2 T_{\max }$ (vertical dotted lines). (b) $P^{\mathrm{L}}$ as a function of pulse numbers $n$ for pulse intervals in the vicinity of $T_{\max }=330 \mathrm{~ms}$ and fixed $E=1 \mathrm{~V} / \mathrm{cm}$. For $T \geq$ $T_{\max }, P^{\mathrm{L}}$ monotonically increases with $n$, while for $T=300 \mathrm{~ms}, n=4$ is optimal. (c) $P^{\mathrm{L}}$ as a function of $E$ for different $n$ and fixed $T=330 \mathrm{~ms}$. LEAP becomes operative at $E$ about $0.7 \mathrm{~V} / \mathrm{cm}$. (d) $P^{\mathrm{L}}$ as a function of the rescaled field strength $\tilde{E} \equiv \sqrt{n} E$. The lines for $n=5$ and $n=6$ first exceed $90 \%$ termination probability. We conclude that the optimal LEAP protocol, which minimized total energy, consists of 5 or 6 pulses.

Fig. 2. Furthermore, pulse intervals with $T_{\max } / 2$ and $2 T_{\max }$ may also be chosen for LEAP albeit they result in a smaller success probability.

Naturally, the termination probability increases with the number of pulses, except for intervals $T$ slightly below $T_{\max }$, where $n=4$ is optimal, see Fig. 5(b). In this regime, the later pulses seem to reinitiate fibrillation after successful termination. Choosing the optimal interval $T_{\max }$, the minimal field strengths for a LEAP success probability of $90 \%$ are $E_{90}^{\mathrm{L}} \approx$ $0.85 \mathrm{~V} / \mathrm{cm}$ and $E_{90}^{\mathrm{L}} \approx 0.76 \mathrm{~V} / \mathrm{cm}$ for five and six pulses, respectively, see Fig. 5(c).

The overall energy delivered by a LEAP protocol is proportional to the number $n$ of pulses and the square of the electric field strength $E$. Therefore, we define the rescaled field strength by $\tilde{E} \equiv \sqrt{n} E$ such that reduction of total energy is achieved if $\tilde{E}_{90}^{\mathrm{L}}<E_{90}^{\mathrm{bi}}$. For both five and six pulses, a rescaled field strength of $\tilde{E}_{90}^{\mathrm{L}} \approx 1.9 \mathrm{~V} / \mathrm{cm}$ is achieved, see
Fig. 5(d). Applying seven pulses instead, only marginally decreases $E_{90}^{\mathrm{L}}$ and increases the rescaled field strength to $\tilde{E}_{90}^{\mathrm{L}} \approx 2.0 \mathrm{~V} / \mathrm{cm}$. For comparison, the field strength required for a single defibrillation pulse is $E_{90}^{\mathrm{bi}} \approx 5.0 \mathrm{~V} / \mathrm{cm}$, i.e., the achieved total reduction of dissipated energy is about $86 \%$ for five or six pulses, while the reduction per pulse is about $97 \%$ for five pulses and about $98 \%$ for six pulses.

For the optimal pulse interval $T_{\max }$, the termination probability $P^{\mathrm{L}}$ decreases for electric field strengths above $2.8 \mathrm{~V} / \mathrm{cm}$, see Fig. 6(a). In this regime, the later pulses reinitiate fibrillation after successful termination, similar to the situation depicted by the blue line in Fig. 5(b). In fact, the optimal pacing period does not match $T_{\max }$ which is given by the dominant period of the electrical turbulence in the absence of pacing but is shifted to about $365 \mathrm{~ms}$ for $E=3 \mathrm{~V} /$ $\mathrm{cm}$, see Fig. 6(b). With this pacing period, which corresponds to $10 \%$ underdrive pacing, $E_{90}^{\mathrm{L}}$ is increased significantly to about $2.2 \mathrm{~V} / \mathrm{cm}$ for six pulses, see Fig. 6(c), and the energy reduction is only $80 \%$ per pulse, resulting in no reduction of the total energy.

\section{b. LEAP for the (FK) model}

Here, the achieved energy reduction is modest when compared to the (LR) model, see Fig. 7(c). We conjecture that this is due to the broader spectrum of the (FK) model. Again, the pulse interval $T$ is crucial for LEAP success, but the optimal $T_{\max }$ is less defined compared to the (LR) model, see Fig. 7(a). Instead of pacing with the dominant period $\tau_{\mathrm{FK}}, T$ may be increased by about $30 \%$ for a similar success probability. Analogous to the (LR) model, increasing the a)

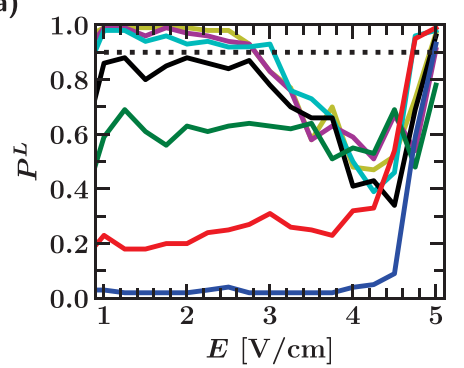

b)

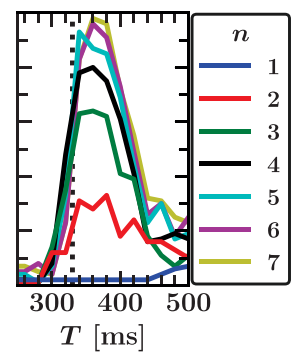

c)

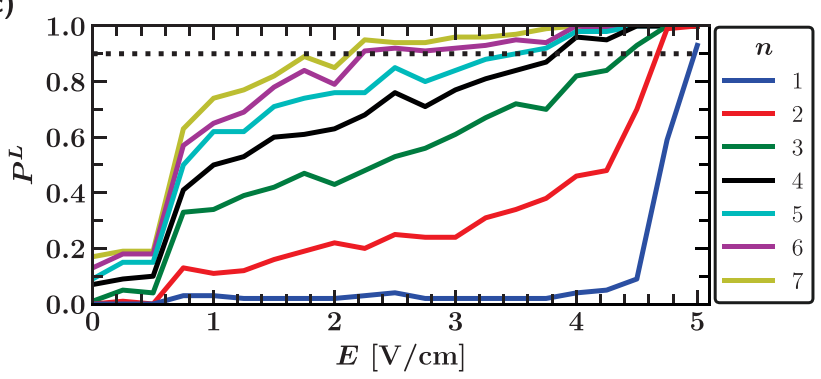

FIG. 6. Termination probability $P^{\mathrm{L}}$ of a suboptimal version of LEAP for the (LR) model and different pulse numbers $n$. (a) $P^{\mathrm{L}}$ as a function of (a relatively high) electric field strength $E$ for $T$ fixed at the dominant period $\tau_{\mathrm{LR}}=$ $330 \mathrm{~ms}$. The termination probability decreases for $E \geq 2.8 \mathrm{~V} / \mathrm{cm}$, since in this regime, the later pulses reinitiate fibrillation after successful termination. (b) $P^{\mathrm{L}}$ as a function of pulse interval $T$ for fixed $E=3 \mathrm{~V} / \mathrm{cm}$. The vertical dotted line indicates the dominant period $\tau_{\mathrm{LR}}=330 \mathrm{~ms}$. (c) $P^{\mathrm{L}}$ as a function of $E$ for different $n$ and fixed $T=365 \mathrm{~ms}$. 
a)

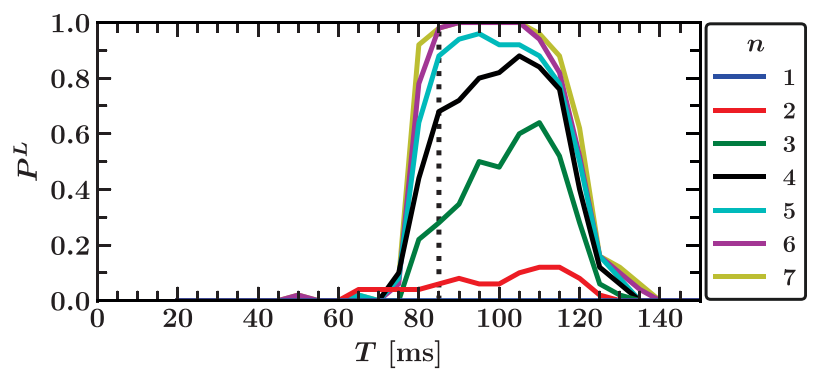

b)

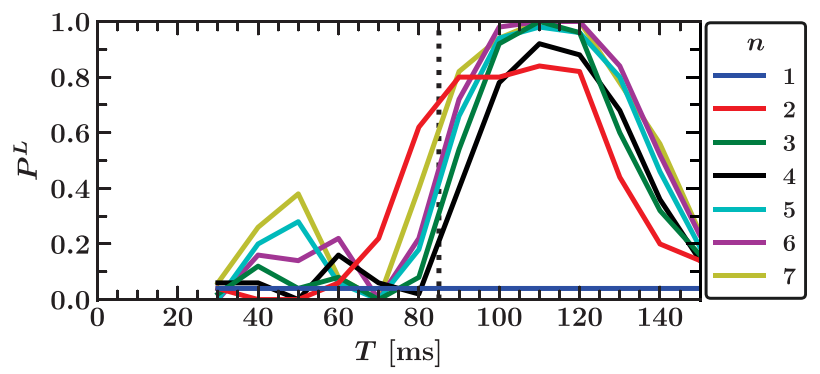

c)

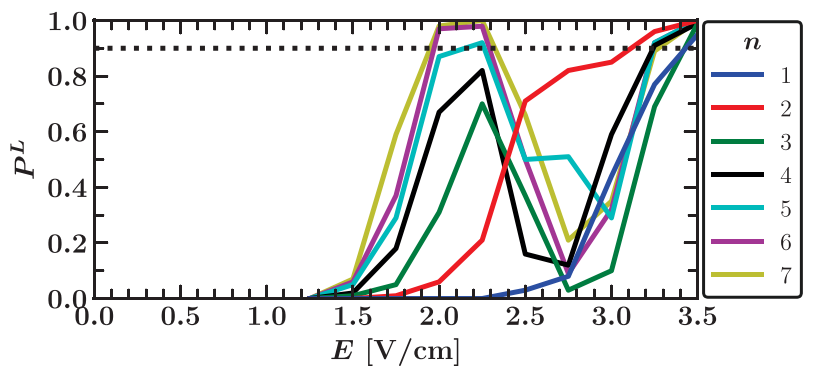

FIG. 7. Termination probability $P^{\mathrm{L}}$ of LEAP for the (FK) model and different pulse numbers $n$. (a) $P^{\mathrm{L}}$ as a function of pulse interval $T$ for fixed field strength $E=2 \mathrm{~V} / \mathrm{cm}$. The dashed vertical line indicates the dominant period $\tau_{\mathrm{FK}}=85 \mathrm{~ms}$. (b) Suboptimal version of LEAP: $P^{\mathrm{L}}$ as a function of pulse interval $T$ for fixed field strength $E=2.5 \mathrm{~V} / \mathrm{cm}$. (c) $P^{\mathrm{L}}$ as a function of $E$ for $T$ fixed at the dominant period $\tau_{\mathrm{FK}}=85 \mathrm{~ms}$.

field strength $E$ results in less termination success, if the pacing period $T$ is unaltered, see Fig. 7(c), since the optimal $T_{\max }$ is increased, see Fig. 7(b).

\section{Summary}

We have performed extensive simulations of electrical turbulence, standard single pulse defibrillation, and LEAP employing both the Luo-Rudy ${ }^{30}$ (LR) and Fenton-Karma ${ }^{31}$ (FK) models. For each set of parameters, simulations were repeated with 100 initial conditions for the electrically turbulent state in order to determine the probability of wave termination. The length scale of both models was chosen such as to reproduce the correct order of magnitude for the number of phase defects of electrical turbulence in cardiac systems and to ensure persistence of turbulence for a reasonable time. Heterogeneities obeying a size distribution typical for blood vessels in cardiac tissue were added and acted as virtual electrodes, i.e., centers for localized tissue activations during defibrillation. The length scale of this distribution was validated by comparing single pulse defibrillation thresholds to experimental values.
Our results are summarized in Table I. Electrical turbulence, i.e., fibrillation, exhibits a frequency spectrum with a distinct peak. For the (LR) model, this peak is relatively narrow compared to the (FK) model. Naturally, fibrillation exhibits less persistence for no-flux boundary conditions. Furthermore, for given boundary conditions and simulation domains, electrical turbulence exhibits different persistencies in different models. However, for both models and periodic boundaries, the half-life $t_{1 / 2}$ of fibrillation is longer than the duration of a defibrillation protocol.

LEAP does operate in simulations with the (LR) model and the $(\mathrm{FK})$ model, but less energy reduction is achieved for the latter. In both cases, optimal results are found for five or six pulses and a pulse interval matching the dominant period of turbulence.

\section{DISCUSSION}

A critical matter of many studies in cardiac modeling is the appropriate choice of length scales. We are confident in the feasibility of our scaling, since it results in a reasonable order of magnitude for both the number of defects and the half-life of unperturbed turbulence. Furthermore, the sizes of the heterogeneities which are observed ${ }^{13}$ match this scale, since it sets the single pulse defibrillation threshold to the experimentally verified values. ${ }^{39}$ In other studies, length scales of geometrically detailed models have been artificially set by decreasing tissue conductivities in order to sustain

TABLE I. Summary of electrical turbulence, single pulse defibrillation, and low-energy antifibrillation pacing (LEAP) probed with the Luo-Rudy (LR) and scaled Fenton-Karma (FK) models. Electrical turbulence is characterized by the average number of defects, the relative width $\delta f / f$ of the main spectral peak, see Fig. 2, and the half-life $t_{1 / 2}$ of its persistence with periodic and no-flux boundaries, see Fig. 3. $E_{90}^{\text {mono }}$ and $E_{90}^{\text {bi }}$ denote the minimal field strength of single mono- and biphasic pulse, respectively, to achieve a $90 \%$ termination probability. Similarly, $E_{90}^{\mathrm{L}}$ is the minimal field strength for LEAP with $n=5,6$, and 7 pulses and $\tilde{E}_{90}^{\mathrm{L}} \equiv \sqrt{n} E_{90}^{\mathrm{L}}$ is the corresponding rescaled quantity, see Fig. 5(d). LEAP needs less energy in total than a single defibrillation pulse if $\tilde{E}_{90}^{\mathrm{L}}<E_{90}^{\mathrm{bi}}$ which is the case for the (LR) model but not for the (FK) model. In particular, five and six pulses spaced by the optimal pulse interval $T_{\max }$ result in $\tilde{E}_{90}^{\mathrm{L}} \approx 1.9 \mathrm{~V} / \mathrm{cm}$ which corresponds to energy reduction of about $86 \%$ in total.

\begin{tabular}{lccc}
\hline \hline & & LR & FK \\
\hline Electrical turbulence & Av. \# defects & 10.4 & 9.6 \\
& $\delta f / f_{\max }$ & 0.146 & 0.44 \\
& $t_{1 / 2}(\mathrm{~s})$ & 9.2 (periodic) & $>10$ (periodic) \\
& & 1.7 (no-flux) & 1.6 (no-flux) \\
Single pulse & $E_{90}^{\text {mono }}(\mathrm{V} / \mathrm{cm})$ & 12.1 (periodic) & 4.4 (periodic) \\
defibrillation & & 12.4 (no-flux) & 4.0 (no-flux) \\
& $E_{90}^{\text {bi }}(\mathrm{V} / \mathrm{cm})$ & 5.0 (periodic) & 3.8 (periodic) \\
& & $5.0($ no-flux) & $3.6($ no-flux $)$ \\
LEAP & $E_{90}^{\mathrm{L}}(\mathrm{V} / \mathrm{cm})$ & $0.85(\mathrm{n}=5)$ & $2.2(\mathrm{n}=5)$ \\
& & $0.76(\mathrm{n}=6)$ & $2.0(\mathrm{n}=6)$ \\
& & $0.74(\mathrm{n}=7)$ & $1.9(\mathrm{n}=7)$ \\
& $\tilde{E}_{90}^{\mathrm{L}}(\mathrm{V} / \mathrm{cm})$ & $1.9(\mathrm{n}=5)$ & $4.8(\mathrm{n}=5)$ \\
& & $1.9(\mathrm{n}=6)$ & $4.8(\mathrm{n}=6)$ \\
& $2.0(\mathrm{n}=7)$ & $5.1(\mathrm{n}=7)$ \\
& & $97 \%(\mathrm{n}=5)$ & $66 \%(\mathrm{n}=5)$ \\
& Energy reduction & $98 \%(\mathrm{n}=6)$ & $72 \%(\mathrm{n}=6)$ \\
& per pulse & $98 \%(\mathrm{n}=7)$ & $75 \%(\mathrm{n}=7)$ \\
\hline \hline
\end{tabular}


arrhythmias, see, e.g., Ref. 29. In the latter study, overdriving VF with $88 \%$ of the dominant cycle length in an anatomically realistic model is reported to result in an energy reduction of $99.4 \%$ per pulse, but this conclusion is drawn from few simulations.

We found that the pacing period for which LEAP works most efficiently is roughly the dominant period of the electrical turbulence. Protocols with multiples and divisors of this optimal period seem to have relatively high success rates too, indicating that the defibrillation process can be understood as a synchronization process. For the (LR) model, a LEAP protocol employing resonant pacing with the mentioned dominant period and five pulses required about $97 \%$ less energy per pulse and $86 \%$ less total energy than a single biphasic shock for successful defibrillation. For the (FK) model, we find an energy reduction of $66 \%$ per pulse, resulting in no reduction of total energy.

The optimal pacing we found is somewhat different from the over- and underdrive pacing applied in LEAP experiments. ${ }^{13}$ For the (LR) model and a field strength of $E=1 \mathrm{~V} / \mathrm{cm}$, employing a pulse interval of $90 \%$ or $110 \%$ of the dominant cycle length reduces the success probability to about 50\% in our simulations, see Fig. 5(a). For larger electric field strengths, however, the optimal pacing period is slightly increased. With $E=3 \mathrm{~V} / \mathrm{cm}$, the optimal pacing period is at about $110 \%$ of the dominant period of electrical turbulence, see Fig. 6(b). This effect can be interpreted as an increased dominant period of the perturbed system: Tissue which gets excited by strong electrical fields has an increased refractory period. Underpacing with a pulse interval of $T=1.1 \tau_{\mathrm{LR}}$ results in an energy reduction of about $80 \%$ per pulse, see Fig. 6(c), but no total energy reduction. We might speculate that the experimental finding ${ }^{13}$ of an energy reduction between $80 \%$ and $90 \%$ per pulse and successful underdrive pacing might correspond to a similar variant of LEAP. A common mechanism is supported by simulations employing the (FK) model which exhibit a similar increase in the optimal pacing period with field strength, see Fig. 7.

\section{CONCLUSION}

We have carried out a statistical study of success probabilities for LEAP with two alternative models of cardiac tissue perforated by blood vessels. In both cases, pacing with the dominant period of electrical turbulence results in maximal energy reduction. For a model featuring a pronounced dominant period, the reduction exceeds $98 \%$ per pulse compared to single pulse defibrillation. If the necessary field strength for LEAP is overestimated, underdrive pacing is superior to resonant pacing. We speculate that such a less efficient protocol was employed in previous LEAP experiments and suggest to test our results with respect to the optimal driving period in future experiments.

\section{SUPPLEMENTARY MATERIAL}

See supplementary material for movies of the simulations. In all movies, the simulation domain has an edge length of about nine times the correlations length $\xi$ and periodic boundary conditions. In movies $4-6$, the (first) pulse is applied at $t=500 \mathrm{~ms}$. All biphasic pulses are applied for $7 \mathrm{~ms}$ in the forward direction and $3 \mathrm{~ms}$ in the backward direction.

Movie 1: Electrical turbulence for (LR) model.

Movie 2: Electrical turbulence for (FK) model.

Movie 3: Defibrillation with single biphasic pulse for (LR) model and $E=5 \mathrm{~V} / \mathrm{cm}$.

Movie 4: Defibrillation with single biphasic pulse for (FK) model and $E=4 \mathrm{~V} / \mathrm{cm}$.

Movie 5: LEAP with five biphasic pulse for (LR) model, $E=0.75 \mathrm{~V} / \mathrm{cm}$ and pulse interval $T=\tau_{\mathrm{LR}}=330 \mathrm{~ms}$.

Movie 6: LEAP with five biphasic pulse for (FK) model, $E=2 \mathrm{~V} / \mathrm{cm}$ and pulse interval $T=\tau_{\mathrm{FK}}=85 \mathrm{~ms}$.

\section{ACKNOWLEDGMENTS}

We acknowledge financial support by DFG through SFB910 (M.B., S.A., and T.N.) and through GRK1558 (P.B. and M.B.). We thank Rodrigo Weber dos Santos, Flavio Fenton, Ulrich Parlitz, Valentin Krinsky, and the anonymous reviewer for discussions.

${ }^{1}$ E. M. Cherry, F. H. Fenton, and R. F. Gilmour, "Mechanisms of ventricular arrhythmias: A dynamical systems-based perspective," Am. J. Physiol. 302, H2451-H2463 (2012).

${ }^{2}$ S. V. Pandit and J. Jalife, "Rotors and the dynamics of cardiac fibrillation," Circ. Res. 112, 849-862 (2013).

${ }^{3}$ A. Karma, "Physics of cardiac arrhythmogenesis," Annu. Rev. Condens. Matter Phys. 4, 313-337 (2013).

${ }^{4}$ Z. Qu, G. Hu, A. Garfinkel, and J. N. Weiss, "Nonlinear and stochastic dynamics in the heart," Phys. Rep. 543, 61-162 (2014).

${ }^{5}$ S. Alonso, M. Bär, and B. Echebarria, "Nonlinear physics of electrical wave propagation in the heart: A review," Rep. Prog. Phys. 79, 096601 (2016).

${ }^{6}$ R. A. Gray, A. M. Pertsov, and J. Jalife, "Spatial and temporal organization during cardiac fibrillation," Nature 392, 75-78 (1998).

${ }^{7}$ F. X. Witkowski, L. J. Leon, P. A. Penkoske, W. R. Giles, M. L. Spano, W. L. Ditto, and A. T. Winfree, "Spatiotemporal evolution of ventricular fibrillation," Nature 392, 78-82 (1998).

${ }^{8}$ M. P. Nash, A. Mourad, R. H. Clayton, P. M. Sutton, C. P. Bradley, M. Hayward, D. J. Paterson, and P. Taggart, "Evidence for multiple mechanisms in human ventricular fibrillation," Circulation 114, 536-542 (2006).

${ }^{9}$ K. H. Ten Tusscher and A. V. Panfilov, "Organization of ventricular fibrillation in the human heart," Circ. Res. 100, e87-e101 (2007).

${ }^{10}$ W. H. Maisel, "Pacemaker and icd generator reliability: Meta-analysis of device registries,” JAMA 295, 1929-1934 (2006).

${ }^{11}$ G. P. Walcott, C. R. Killingsworth, and R. E. Ideker, "Do clinically relevant transthoracic defibrillation energies cause myocardial damage and dysfunction?," Resuscitation 59, 59-70 (2003).

${ }^{12}$ F. H. Fenton, S. Luther, E. M. Cherry, N. F. Otani, V. Krinsky, A. Pumir, E. Bodenschatz, and R. F. Gilmour, "Termination of atrial fibrillation using pulsed low-energy far-field stimulation," Circulation 120, 467-476 (2009).

${ }^{13}$ S. Luther, F. H. Fenton, B. G. Kornreich, A. Squires, P. Bittihn, D. Hornung, M. Zabel, J. Flanders, A. Gladuli, L. Campoy, E. M. Cherry, G. Luther, G. Hasenfuss, V. I. Krinsky, A. Pumir, R. F. Gilmour, Jr., and E. Bodenschatz, "Low-energy control of electrical turbulence in the heart," Nature 475, 235-239 (2011).

${ }^{14}$ C. M. Ambrosi, C. M. Ripplinger, I. R. Efimov, and V. V. Fedorov, "Termination of sustained atrial flutter and fibrillation using low-voltage multiple-shock therapy," Heart Rhythm 8, 101-108 (2011).

${ }^{15}$ W. Li, A. H. Janardhan, V. V. Fedorov, Q. Sha, R. B. Schuessler, and I. R. Efimov, "Low-energy multistage atrial defibrillation therapy terminates atrial fibrillation with less energy than a single shock clinical perspective," Circ.: Arrhythmia Electrophysiol. 4, 917-925 (2011).

${ }^{16}$ W. Li, C. M. Ripplinger, Q. Lou, and I. R. Efimov, "Multiple monophasic shocks improve electrotherapy of ventricular tachycardia in a rabbit model of chronic infarction," Heart Rhythm 6, 1020-1027 (2009). 
${ }^{17}$ A. H. Janardhan, W. Li, V. V. Fedorov, M. Yeung, M. J. Wallendorf, R. B. Schuessler, and I. R. Efimov, "A novel low-energy electrotherapy that terminates ventricular tachycardia with lower energy than a biphasic shock when antitachycardia pacing fails," J. Am. Coll. Cardiol. 60, 2393-2398 (2012).

${ }^{18} \mathrm{~S}$. Weidmann, "Effect of current flow on the membrane potential of cardiac muscle," J. Physiol. 115, 227-236 (1951).

${ }^{19}$ J. P. Wikswo, Jr., S.-F. Lin, and R. A. Abbas, "Virtual electrodes in cardiac tissue: A common mechanism for anodal and cathodal stimulation," Biophys. J. 69, 2195 (1995).

${ }^{20}$ A. Pumir and V. Krinsky, "Unpinning of a rotating wave in cardiac muscle by an electric field," J. Theor. Biol. 199, 311-319 (1999).

${ }^{21}$ A. Pumir, V. Nikolski, M. Hörning, A. Isomura, K. Agladze, K. Yoshikawa, R. Gilmour, E. Bodenschatz, and V. Krinsky, "Wave emission from heterogeneities opens a way to controlling chaos in the heart," Phys. Rev. Lett. 99, 208101 (2007).

${ }^{22} \mathrm{P}$. Bittihn, M. Hörning, and S. Luther, "Negative curvature boundaries as wave emitting sites for the control of biological excitable media," Phys. Rev. Lett. 109, 118106 (2012).

${ }^{23}$ B. J. Caldwell, M. L. Trew, and A. M. Pertsov, "Cardiac response to low energy field pacing challenges the standard theory of defibrillation," Circ.: Arrhythmia Electrophysiol. 8, 685 (2015).

${ }^{24}$ S. Takagi, A. Pumir, D. Pazo, I. Efimov, V. Nikolski, and V. Krinsky, "Unpinning and removal of a rotating wave in cardiac muscle," Phys. Rev. Lett. 93, 058101 (2004).

${ }^{25}$ C. M. Ripplinger, V. I. Krinsky, V. P. Nikolski, and I. R. Efimov, "Mechanisms of unpinning and termination of ventricular tachycardia," Am. J. Physiol.: Heart Circ. Physiol. 291, H184-H192 (2006).

${ }^{26}$ A. Pumir, S. Sinha, S. Sridhar, M. Argentina, M. Hörning, S. Filippi, C. Cherubini, S. Luther, and V. Krinsky, "Wave-train-induced termination of weakly anchored vortices in excitable media," Phys. Rev. E 81, 010901 (2010).

${ }^{27}$ T. Shajahan, S. Berg, S. Luther, V. Krinski, and P. Bittihn, "Scanning and resetting the phase of a pinned spiral wave using periodic far field pulses," New J. Phys. 18, 043012 (2016).

${ }^{28}$ D. Hornung, V. Biktashev, N. Otani, T. Shajahan, T. Baig, S. Berg, S. Han, V. Krinsky, and S. Luther, "Mechanisms of vortices termination in the cardiac muscle," Royal Soc. Open Sci. 4, 170024 (2017).

${ }^{29}$ L. J. Rantner, B. M. Tice, and N. A. Trayanova, "Terminating ventricular tachyarrhythmias using far-field low-voltage stimuli: Mechanisms and delivery protocols," Heart Rhythm 10, 1209-1217 (2013).

${ }^{30}$ L. Luo and Y. Rudy, "A model of the ventricular cardiac action potential: Depolarization, repolarization and their interaction," Circ. Res. 68, 1501-1526 (1991).

${ }^{31}$ F. Fenton and A. Karma, "Vortex dynamics in three-dimensional continuous myocardium with fiber rotation: Filament instability and fibrillation," Chaos 8, 20-47 (1998).
${ }^{32} \mathrm{~K}$. Ten Tusscher and A. Panfilov, "Cell model for efficient simulation of wave propagation in human ventricular tissue under normal and pathological conditions," Phys. Med. Biol. 51, 6141 (2006).

${ }^{33}$ J. P. Keener and J. Sneyd, Mathematical Physiology (Springer, 2009), Vol. 1.

${ }^{34}$ X. Zhou, W. M. Smith, D. L. Rollins, and R. E. Ideker, "Transmembrane potential changes caused by shocks in guinea pig papillary muscle," Am. J. Physiol.: Heart Circ. Physiol. 271, H2536-H2546 (1996).

${ }^{35}$ M. M. Elshrif and E. M. Cherry, "A quantitative comparison of the behavior of human ventricular cardiac electrophysiology models in tissue," PLoS One 9, e84401 (2014).

${ }^{36}$ N. F. Otani, A. Mo, S. Mannava, F. H. Fenton, E. M. Cherry, S. Luther, and R. F. Gilmour, Jr., "Characterization of multiple spiral wave dynamics as a stochastic predator-prey system," Phys. Rev. E 78, 021913 (2008).

${ }^{37} \mathrm{~S}$. Weidmann, "Electrical constants of trabecular muscle from mammalian heart," J. Physiol. 210, 1041-1054 (1970).

${ }^{38}$ A. Bueno-Orovio, E. M. Cherry, and F. H. Fenton, "Minimal model for human ventricular action potentials in tissue," J. Theor. Biol. 253, 544-560 (2008).

${ }^{39}$ F. X. Witkowski, P. A. Penkoske, and R. Plonsey, "Mechanism of cardiac defibrillation in open-chest dogs with unipolar dc-coupled simultaneous activation and shock potential recordings," Circulation 82, 244-260 (1990).

${ }^{40}$ I. R. Efimov, M. W. Kroll, and P. Tchou, Cardiac Bioelectric Therapy: Mechanisms and Practical Implications (Springer Science \& Business Media, 2008).

${ }^{41}$ U. Achleitner, K. Rheinberger, B. Furtner, A. Amann, and M. Baubin, "Waveform analysis of biphasic external defibrillators," Resuscitation 50, 61-70 (2001).

${ }^{42}$ J. Bragard, A. Simic, J. Elorza, R. O. Grigoriev, E. M. Cherry, R. F. Gilmour, Jr., N. F. Otani, and F. H. Fenton, "Shock-induced termination of reentrant cardiac arrhythmias: Comparing monophasic and biphasic shock protocols," Chaos 23, 043119 (2013).

${ }^{43}$ W. Hackbusch, Elliptic Differential Equations: Theory and Numerical Treatment, Springer Series in Computational Mathematics (Springer, 2003).

${ }^{44}$ S. Rush and H. Larsen, "A practical algorithm for solving dynamic membrane equations," IEEE Trans. Biomed. Eng. 25, 389-392 (1978).

${ }^{45}$ E. Isaacson and H. Keller, Analysis of Numerical Methods, Dover Books on Mathematics Series (Dover Publications, 1994).

${ }^{46}$ A. Winfree, "Evolving perspectives during 12 years of electrical turbulence," Chaos 8, 1-19 (1998).

${ }^{47}$ A. Wacker, S. Bose, and E. Schöll, "Transient spatio-temporal chaos in a reaction-diffusion model," Europhys. Lett. 31, 257 (1995).

${ }^{48}$ M. C. Strain and H. S. Greenside, "Size-dependent transition to highdimensional chaotic dynamics in a two-dimensional excitable medium," Phys. Rev. Lett. 80, 2306 (1998).

${ }^{49}$ R. Wackerbauer and K. Showalter, "Collapse of spatiotemporal chaos," Phys. Rev. Lett. 91, 174103 (2003). 